MRI and 1H-MRS may prove effective in assessment of effects of cholesterol replacement therapy in patients with SLOS (Caruso PA et al. Neuroradiology 2004 Jan;46(1):3-14). Of 18 patients with SLOS, abnormal CNS findings were noted in 5 patients, including callosal abnormalities in $4(22 \%)$, Dandy-Walker variant in 1 , arachnoid cyst in 1 , and holoprosencephaly in $1(6 \%)$. Clinical degree of disease severity was correlated with lipid:choline ratios.

\title{
DEVELOPMENTAL OUTCOME FOLLOWING HEMISPHERECTOMY FOR HEMIMEGALENCEPHALY
}

Investigators at the National Center of Neurology and Psychiatry, Tokyo, studied the effect of early hemispherectomy on development in a consecutive series of 12 infants with hemimegalencephaly (HME) and epileptic encephalopathy. Mean age at onset was 20.4 days, mean age at surgery was 4.3 months (range 2-9), and mean follow-up time was 78.8 months (36-121). Eleven patients had a history of early infantile epileptic encephalopathy. Following vertical parasagittal hemispherectomy, 8 (66.7\%) were seizure-free and showed significantly higher postoperative developmental quotient (DQ) than those with seizures (mean 31.3 vs $5.5 ; \mathrm{p}=0.02$ ). In the seizure-free group, postoperative DQ correlated with postoperative seizure duration $(p=0.01)$. Shorter seizure duration during early infancy provides better postoperative DQ in infants with HME and epileptic encephalopathy. (Honda R, Kaido T, Sugai K, et al. Long-term developmental outcome after early hemispherectomy for hemimegalencephaly in infants with epileptic encephalopathy. Epilepsy Behav 2013 Aug 6;29(1):30-35). (Response: Dr Kaido. Email: kaido@ncnp.go.jp).

COMMENT. Despite the risks, the authors conclude that early surgical intervention for hemimegalencephaly is preferable to brain damage from repeated seizures and encephalopathy and long-term AED use. A similar conclusion was reached by investigators at the Sorbonne, Paris, France, who reviewed the literature and reports of $\sim 600$ cases in the last 30 years (Bulteau C et al. Epilepsy surgery for hemispheric syndromes in infants: hemimegalencephaly and hemispheric cortical dysplasia. Brain Dev 2013 Sep;35(8):742-7). Hemispheric surgical procedures are considered safe and can be performed from the first month of life. Residual insular cortex and contralateral MRI abnormalities are associated with persistent postoperative seizures and lack of cognitive improvement.

\section{NEUROCUTANEOUS DISORDERS}

\section{mTOR INHIBITION AND TUBEROUS SCLEROSIS PREVENTION}

Investigators at Children's Memorial Health Institute, Warsaw, Poland, report monozygotic twin sisters with tuberous sclerosis complex (TSC), one treated with the mTOR inhibitor everolimus since age 4 years. After 24-month follow-up, everolimus treatment was associated with a significant decrease in brain tumor volume, and the treated twin presents no facial angiofibroma, and no renal angiomyolipomas (AMLs). 